\title{
EVALUACIÓN DE LÍNEAS DE UNA CRUZA INTERESPECÍFICA DE TOMATE
}

\section{EVALUATION OF INBRED LINES FROM A TOMATO INTERSPECIFIC CROSS}

\author{
Mayra A. Parra-Gómez¹, Ricardo Lobato-Ortiz*, J. Jesús García-Zavala', \\ Delfino Reyes-López ${ }^{2}$ y Mario J. Velasco-Alvarado'
}

\begin{abstract}
'Postgrado en Recursos Genéticos y Productividad-Genética, Campus Montecillo, Colegio de Postgraduados. Km. 36.5 Carr. México-Texcoco. 56230, Montecillo, Texcoco, Edo. de México. Tel. (595) 20200 Ext. 1534. ²Facultad de Ingeniería Agrohidráulica, Benemérita Universidad Autónoma de Puebla. San Juan Acateno, Teziutlán, Puebla.

*Autor para correspondencia (rlobato@colpos.mx)
\end{abstract}

\section{RESUMEN}

El tomate 0 jitomate (Solanum lycopersicum L.) es una de las principales hortalizas cultivadas en México y en el mundo; sin embargo, presenta una reducida variación genética resultado de su proceso de domesticación y de su sistema de reproducción por autogamia. Como consecuencia, el uso de germoplasma proveniente de sus parientes silvestres es una opción que los mejoradores han explotado para buscar variantes alélicas de calidad y de resistencia a factores bióticos y abióticos adversos. El objetivo de este trabajo fue ampliar la base genética del jitomate cultivado, generar germoplasma para el mejoramiento genético y evaluar el potencial hortícola de 225 líneas $\mathrm{S}_{3}$ derivadas de una cruza interespecífica entre $\mathrm{S}$. lycopersicum y $\mathrm{S}$. pimpinellifolium. Las 225 líneas se evaluaron en condiciones de invernadero e hidroponía. Se obtuvo gran variación entre líneas para las variables relacionadas con el rendimiento y sus componentes, así como en calidad de fruto; el peso promedio de fruto varió de 1 a $36 \mathrm{~g}$, los sólidos solubles totales de 3.4 a $10.2^{\circ}$ Brix, el rendimiento de 16 a $1330 \mathrm{~g}$ por planta, y el número de frutos por racimo fue de 8 a 115, por lo que las líneas generadas representan una nueva fuente de germoplasma para programas de mejoramiento genético de jitomate en México. Las variables que presentaron alta correlación con el rendimiento fueron el peso promedio de fruto, longitud y diámetro de fruto, así como el número total de frutos por planta. Los componentes del rendimiento tuvieron valores relativamente altos de heredabilidad en sentido amplio, mayores de 0.60 , mientras que para grados sólidos solubles totales fue 0.50 .

Palabras clave: Solanum lycopersicum, Solanum pimpinellifolium, cruzas interespecífícas, mejoramiento genético.

\section{SUMMARY}

Tomato (Solanum lycopersicum L.) is one of the main vegetables grown in México and around the world; however, this species has reduced genetic variation due to its domestication and inbreeding. As a consequence tomato breeders use wild germplasm from tomato relatives in search ing allelic variants for fruit quality and resistance to adverse biotic and abiotic factors. This work tried to increase the genetic background of cultivated tomato, to generate germplasm for tomato breeding in México, and to assess the horticultural potential of 225 recombinant $\mathrm{S}_{3}$ lines derived from an interspecific cross between S. lycopersicum and S. pinpinellifolium. The 225 lines were evaluated under greenhouse and hydroponics conditions. Large variation was found among lines for yield and yield components, as well as for fruit quality. For example, average fruit weight ranged from $1 \mathrm{~g}$ to $36 \mathrm{~g}$; total soluble solids ranged from 3.4 to $10.2^{\circ} \mathrm{Brix}$; fruit yield ranged from 16 to $1330 \mathrm{~g}$ per plant; and the number of fruits per cluster ranged from 8 to 115 . Therefore the lines generated in this work represent a new source of germplasm for tomato breeding programs in México. Traits that showed higher correlation with yield were the variable averages of fruit weight, fruit length, fruit diameter, and total number of fruit per plant. Yield components had relatively high values of broad sense heritability, greater than 0.60 , while for total soluble solids it was 0.50 .

Index words: Solanum lycopersicum, Solanum pimpinellifolium, interspecific crosses, plant breeding.

\section{INTRODUCCIÓN}

El tomate o jitomate (Solanum lycopersicum L.) es una de las principales hortalizas cultivadas en México y el mundo. La producción nacional de jitomate en el año 2014 fue de 2.8 millones de toneladas (SIAP, 2015). Esta especie es originaria de la planicie costera occidental de América del Sur, pero se considera a México como su centro de domesticación (Jenkins, 1948; Rick y Forbes, 1975; Peralta y Spooner, 2007).

En cuanto a su diversidad, dentro de la sección Lycopersicon se incluyen los 12 parientes silvestres más relacionados con el jitomate cultivado (Peralta et al., 2008), y se considera a S. pimpinellifolium como el pariente más cercano al tipo cultivado (Miller y Tanksley, 1990). Las diferencias genéticas ocurren de manera natural entre los organismos dentro de las poblaciones y en las especies, pero la variación genética del jitomate cultivado es reducida, ya que solo posee $4.48 \%$ de la variación existente en sus parientes silvestres (Miller y Tanksley, 1990). El mejoramiento genético requiere diversidad alélica o una base genética amplia para explorar rasgos deseables y desarrollar variedades con características novedosas (Canady et al., 2006).

Por esta razón, los fitomejoradores exploran nuevas fuentes de germoplasma de parientes silvestres para utilizarlas en el mejoramiento de la especie. Entre las especies emparentadas con el jitomate, numerosas accesiones de 
especies silvestres se han empleado en la introgresión de genes de resistencia a factores estresantes del desarrollo de la especie cultivada (Dueñas, 2009). S. pimpinellifolium (Lippman y Tanksley, 2001) es una fuente atractiva de germoplasma para el mejoramiento de variedades modernas por medio de cruzas interespecíficas con S. lycopersicum.

Aun cuando el uso de parientes silvestres del jitomate en el fitomejoramiento es frecuente en varios países desde hace varios años, en México son escasos este tipo de trabajos (Hernández-Bautista et al., 2014). Por ello es necesario realizar trabajos para explorar y evaluar la capacidad productiva de nuevos genotipos generados a partir de cruzas interespecíficas de jitomate, que permitan hacer uso de tal variación, como se ha hecho en otros países (Foolad, 2007). Los objetivos del presente trabajo fueron ampliar la base genética del jitomate cultivado, generar germoplasma para el mejoramiento genético en México, y evaluar el potencial de líneas $\mathrm{S}_{3}$ derivadas de una cruza interespecífica entre S. lycopersicum y S. pimpinellifolium.

\section{MATERIALES Y MÉTODOS}

\section{Material vegetal y sitio experimental}

El material vegetal consistió de 225 líneas $S_{3}$ derivadas de una población proveniente de la cruza interespecífica entre Solanum lycopersicum, una línea derivada de la colecta LOR82 del tipo "chino criollo" (Bonilla-Barrientos et al., 2014) de Tehuacán, Puebla, Méx., y una línea proveniente de la especie silvestre Solanum pimpinellifolium, colecta 11904. Las líneas $S_{3}$ se derivaron mediante el método "selección uniseminal" (Márquez, 1988). Además, se incluyó al híbrido Súper Sweet 100 como testigo. El experimento se estableció en invernaderos de plástico ubicados en Montecillo, Texcoco, Estado de México ( $19^{\circ} 30^{\prime \prime}$ N y $98^{\circ} 53^{\prime \prime}$ $\mathrm{O}$, a una altitud de $2250 \mathrm{~m}$ ). El cultivo se estableció durante el periodo de primavera - verano del año 2013.

\section{Diseño experimental y manejo agronómico}

El experimento se estableció en un diseño de bloques completos al azar con dos repeticiones, con cuatro plantas por repetición. El trasplante se realizó el día 10 de mayo del año 2013, 35 d después de la siembra, en bolsas de polietileno de color negro de $40 \times 40 \mathrm{~cm}$ con tezontle rojo fino como sustrato, a una densidad de 25,000 plantas ha-1. Durante el primer mes posterior al trasplante (estado vegetativo) se aplicó la solución nutritiva propuesta por Steiner (1984), a una concentración de 50 \%; una vez que las plantas iniciaron la etapa de floración, la concentración se incrementó a $100 \%$. El pH de la solución se mantuvo entre 5.5 y 6.0. Durante el ciclo de cultivo se realizaron aplicaciones preventivas de fungicidas y plaguicidas, como Cap- $\tan \circledast$ (captan), Confidor $\AA^{(i m i d a c l o p r i d), ~ B e l e a f ® ~(f l o n i c a-~}$ mid), Cupravit ${ }^{\circledR}$ (oxicloruro de cobre), Ampligo ${ }^{\circledR}$ (lambda cyalotrina y clorantraniliprol), para el control de mosca blanca (Bemisia tabaci Gennadius) (Hemiptera, Aleyrodidae), paratrioza (Bactericera cockerelli), y tizón tardío (Phytophthora infestans).

\section{Variables registradas}

Las variables evaluadas fueron altura de planta $(\mathrm{cm})$, medida de la base del tallo al ápice a los 90 d; número de flores del tercer racimo, número de racimos totales a los $90 \mathrm{~d}$; número total de frutos por planta y peso total de frutos por planta $(\mathrm{g})$ se tomaron al final de la cosecha que consistió en dos cortes; peso promedio de fruto (g), diámetro de fruto $(\mathrm{mm})$, longitud del fruto $(\mathrm{mm})$, número de lóculos y sólidos solubles totales ('Brix) se registraron en una muestra de cinco frutos maduros por planta. Los sólidos solubles totales se registraron en porcentaje y fueron medidos con un refractómetro digital (marca ATAGO®, modelo PAL-1; México).

\section{Análisis de datos}

En cada variable se realizó un análisis de varianza y una comparación de medias mediante la prueba de Tukey ( $\mathrm{P} \leq$ 0.05) con el paquete estadístico SAS v.9.0 (SAS Institute, 2002). Luego se hizo un análisis de correlación para determinar la relación existente entre las variables. Finalmente, se estimó la heredabilidad en sentido amplio $(H)$ de las variables (Holland et al., 2003).

\section{RESULTADOS Y DISCUSIÓN}

\section{Análisis de varianza}

Hubo diferencias altamente significativas entre genotipos (GEN) para las 10 variables (Cuadro 1), como consecuencia de la variación entre las líneas, atribuible a que provienen de la cruza de la especie silvestre y la cultivada, por lo que se esperaba una gran variación tanto en la F2 como en las generaciones avanzadas de dicha cruza.

Los mayores coeficientes de variación se observaron en las variables número total de frutos (TF) con $41.4 \%$, peso total de fruto por planta (PTP) con $40.7 \%$, flores por racimo (FR) con $31.9 \%$ y peso promedio del fruto (PPF) con $31.6 \%$. El resto de los valores fueron menores a $15 \%$; los coeficientes más bajos fueron para las variables altura de planta (ALT) con $8.5 \%$, largo promedio de fruto (LPF) y diámetro promedio de fruto (DPF) con $10.6 \%$ y $11 \%$, respectivamente. Estos coeficientes de variación son relativamente más altos que los reportados por Carrillo-Rodríguez et al. (2013), quienes al estudiar la variación estacional en 
poblaciones nativas de jitomate encontraron CV menores de $21 \%$ para rendimiento/planta, y de $23 \%$ para el número de frutos por racimo. Lo anterior podría deberse a que las líneas avaluadas en este trabajo están todavía segregando para muchos loci, ya que son líneas $S_{3}$ provenientes de una cruza interespecífica.

\section{Comparación de medias}

En el Cuadro 2 se presenta la comparación de medias de las variables para las 10 líneas $\mathrm{S}_{3}$ sobresalientes, así como para la línea de más bajo desempeño y el testigo comercial (Súper Sweet 100). Los genotipos sobresalientes para total de frutos (TF) fueron las líneas 93, 92, 66 y 7, las cuales estuvieron incluidas en el grupo estadístico superior, con 250, 203, 190 y 157 frutos, respectivamente, valores estadísticamente superiores al del testigo que tuvo 96 frutos por planta. En cuanto a rendimiento por planta (PTP), las líneas 189, 187, 236, 83, 136 y 128 tuvieron los mayores rendimientos, los cuales variaron de 730 a $1330 \mathrm{~g} /$ planta, mientras que el testigo rindió $541 \mathrm{~g} /$ planta.

En ${ }^{\circ}$ Brix, las 10 mejores líneas estuvieron en el grupo estadístico superior con valores que fluctuaron de 8.9 a 10.2, mientras que el testigo tuvo un valor de 5.9. Los valores obtenidos en las líneas $S_{3}$ para las características antes señaladas fueron superiores a los del testigo, por lo que estas líneas representan una importante fuente de germoplasma para incrementar los sólidos solubles totales, además de ampliar la base genética del jitomate cultiva- do. Estos resultados eran de esperarse, pues los frutos del progenitor silvestre S. pimpinellifolium tienen altos contenidos de sólidos solubles (Hernández-Bautista et al., 2014). Los $^{\circ}$ Brix constituyen un importante criterio de calidad, que en los jitomates tipo "saladette" varían de 3.9 a 5.2 (Hernández-Leal et al., 2013) mientras que en el germoplasma nativo se han reportado valores de 3.8 a 8.3 (Crisanto-Juárez et al., 2010), y de 5.8 a 8.0 (Juárez- López et al., 2009). En colectas de germoplasma nativo mexicano Bonilla-Barrientos et al. (2014) encontraron contenidos promedio de sólidos solubles totales de 3.8 a $4.4^{\circ} \mathrm{Brix}$.

En contraste, las líneas de más bajo desempeño listadas al final del Cuadro 2, fueron: línea 132 con 8.0 frutos por planta; línea 133 con $16.0 \mathrm{~g}$ de PTP; línea 210 con $1.0 \mathrm{~g}$ por fruto, y línea 223 con $3.4^{\circ}$ Brix. Los valores anteriores comparados con los valores de las líneas de mayor desempeño ponen de manifiesto la gran amplitud de la variación genética que existe entre los genotipos recombinantes derivados de la cruza de S. lycopersicum x S. pimpenillifolium (Hernández-Bautista et al., 2014).

\section{Correlaciones entre variables}

La matriz de correlaciones simples de Pearson entre cada par de variables (Cuadro 3) muestra que 21 coeficientes fueron significativos, los cuales representan $47 \%$ del total, 12 de ellos positivos y 9 negativos. Con el rendimiento de fruto por planta (PTP), las variables que estuvieron correlacionadas positivamente fueron: peso promedio de

Cuadro 1. Cuadrados medios del análisis de varianza, coeficientes de variación y valores de heredabilidad de las 10 variables cuantitativas.

\begin{tabular}{crrrrr}
\hline \multicolumn{5}{c}{ Fuentes de variación } \\
\cline { 2 - 6 } & \multicolumn{1}{c}{ GEN } & REP & Error & CV $(\%)$ & Heredabilidad (H) \\
\hline GL & 224 & 1 & 224 & \\
ALT & $1046.3 * *$ & 1486.2 & 250.7 & 8.5 & 0.61 \\
RTP & $6.2 * *$ & 17.6 & 1.5 & 13.4 & 0.50 \\
FFR & $340.6 * *$ & 1.9 & 43.3 & 31.9 & 0.77 \\
TF & $2128.5 * *$ & 477.9 & 490 & 41.4 & 0.62 \\
PTP & $83308.6 * *$ & 3448.8 & 16296.7 & 40.7 & 0.67 \\
PPF & $56.4 * *$ & 18.9 & 6.3 & 31.6 & 0.78 \\
LPF & $43.7 * *$ & 7.6 & 4.6 & 10.6 & 0.79 \\
DPF & $47.3 * *$ & 23.3 & 5.7 & 11.0 & 0.77 \\
SST & $2.9 * *$ & 2 & 0.7 & 13.1 & 0.50 \\
NL & $0.1 * *$ & 0.02 & 0.06 & 11.9 & 0.50 \\
\hline
\end{tabular}

**significativo $(\mathrm{P}<0.01) ; \mathrm{GL}$ = grados de libertad; GEN = genotipos; REP = repeticiones; CV $(\%)$ = coeficiente de variación; $\mathrm{ALT}$ = altura $(\mathrm{cm}) ; \mathrm{RTP}=$ racimos totales por planta; $\mathrm{FR}$ = flores por racimo; TF = total de frutos; $\mathrm{PPT}=$ peso promedio por planta $(\mathrm{g}) ; \mathrm{PPF}=$ peso promedio por fruto $(\mathrm{g})$; $\mathrm{LPF}$ $=$ largo promedio de fruto $(\mathrm{mm}) ; \mathrm{DPF}=$ diámetro promedio de fruto $(\mathrm{mm}) ; \mathrm{SST}=$ sólidos solubles totales $\left({ }^{\circ}\right.$ Brix$) ; \mathrm{NL}=$ número de lóculos. 
Cuadro2. Comparación de medias para ocho variables cuantitativas de las 10 líneas $\mathrm{S}_{3}$ más sobresalientes, el testigo y la línea de más bajo desempeño.

\begin{tabular}{|c|c|c|c|c|c|c|c|}
\hline Línea & ALT & Línea & RTP & Línea & $F R$ & Línea & $\mathrm{TF}$ \\
\hline 38 & 241.2 a & 226 & $15.0 \mathrm{a}$ & 79 & 115.0 a & 93 & 249.9 a \\
\hline 168 & $238.5 a b$ & 7 & $14.5 \mathrm{ab}$ & 166 & 110.8 a & 92 & $203.2 \mathrm{ab}$ \\
\hline 167 & 234.8 a-c & 93 & 13.2 a-c & 145 & $60.8 \mathrm{~b}$ & 66 & 190.7 a-c \\
\hline 188 & $233.3 \mathrm{a}-\mathrm{d}$ & 186 & $13.0 \mathrm{a}-\mathrm{d}$ & 226 & 53.5 bc & 7 & $157.1 \mathrm{a}-\mathrm{d}$ \\
\hline 132 & 228.7 a-e & 112 & $12.9 \mathrm{a}-\mathrm{d}$ & 66 & $52.8 b-d$ & 97 & 133.0 b-e \\
\hline 33 & $227.4 a-f$ & 201 & 12.8 a-e & 92 & $51.0 \mathrm{~b}-\mathrm{e}$ & 88 & $125.4 b-f$ \\
\hline 201 & 226.8 a-g & 66 & 12.8 a-e & 93 & 50.8 b-f & 108 & $123.2 \mathrm{~b}-\mathrm{g}$ \\
\hline 94 & 224.0 a-h & 57 & 12.6 a-f & 26 & $45.9 \mathrm{~b}-\mathrm{g}$ & 102 & 118.9 b-h \\
\hline 192 & $221.3 \mathrm{a}-\mathrm{i}$ & 168 & $12.5 \mathrm{a}-\mathrm{f}$ & 197 & $43.5 b-h$ & 96 & 118.7 b-h \\
\hline 57 & $221.2 \mathrm{a}-\mathrm{I}$ & 227 & 12.0 a-g & 31 & 42.8 b-i & 145 & 115.5 b-i \\
\hline Súper & $168.2 \mathrm{a}-\mathrm{u}$ & Súper & 11.6 a-i & Súper & $11.4 j-p$ & Súper & $96.0 c-j$ \\
\hline 191 & $126.3 \mathrm{~s}-\mathrm{u}$ & 6 & $5.5 \mathrm{j}-1$ & 114 & 7.8 op & 132 & 7.6 jk \\
\hline \multirow[t]{2}{*}{ DMS } & 74.8 & & 5.8 & & 31.1 & & 104.6 \\
\hline & PTP & Línea & PPF & Línea & SST & Línea & $\mathrm{NL}$ \\
\hline 189 & $1330.3 \mathrm{a}$ & 128 & $36.7 \mathrm{a}$ & 210 & $10.2 \mathrm{a}$ & 6 & $3.8 \mathrm{a}$ \\
\hline 187 & $1223.8 a b$ & 29 & $34.9 \mathrm{ab}$ & 54 & $10.1 \mathrm{ab}$ & 23 & $3.4 a b$ \\
\hline 236 & $1043.3 a-c$ & 187 & $32.5 \mathrm{a}-\mathrm{c}$ & 66 & 9.8 a-c & 55 & $3.3 a-c$ \\
\hline 83 & 875.0 a-d & 136 & $27.0 \mathrm{a}-\mathrm{d}$ & 102 & $9.8 \mathrm{a}-\mathrm{c}$ & 9 & $3.3 \mathrm{a}-\mathrm{c}$ \\
\hline 136 & 850.3 a-e & 120 & 24.0 b-e & 49 & 9.7 a-d & 2 & $3.0 a-d$ \\
\hline 128 & 730.0 a-f & 114 & $21.1 \mathrm{c}-\mathrm{f}$ & 68 & 9.6 a-e & 177 & 3.0 a-d \\
\hline 120 & 686.9 b-g & 104 & $20.5 d-g$ & 52 & 9.6 a-e & 53 & $3.0 a-d$ \\
\hline 92 & 686.4 b-g & 189 & 20.0 d-h & 237 & $9.5 a-f$ & 21 & 3.0 a-d \\
\hline 98 & 679.6 b-h & 98 & $19.5 \mathrm{~d}-\mathrm{i}$ & 112 & 8.9 a-g & 41 & 3.0 a-d \\
\hline 224 & 650.0 b-i & 23 & $17.5 \mathrm{~d}-\mathrm{j}$ & 45 & 8.9 a-h & 157 & $3.0 \mathrm{a}-\mathrm{d}$ \\
\hline Súper & $541.5 \mathrm{c}-\mathrm{m}$ & Súper & $9.9 f-x$ & Súper & $5.9 c-p$ & Súper & $2.3 b-d$ \\
\hline 133 & $15.9 \mathrm{Im}$ & 210 & $1.0 x$ & 223 & $3.4 \mathrm{P}$ & 185 & $2.0 \mathrm{~d}$ \\
\hline DMS & 603.6 & & 11.9 & & 4.0 & & 1.2 \\
\hline
\end{tabular}

Medias con la misma letra en una columna no son estadísticamente diferentes ( $\leq 0.05)$; DMS = diferencia mínima significativa; $\mathrm{ALT}=$ altura de planta $(\mathrm{cm}) ; \mathrm{RTP}=$ racimos totales por planta; $\mathrm{FR}=$ flores por racimo; $\mathrm{TF}=$ total de frutos; $\mathrm{PTP}=$ peso total por planta (g); PPF = peso promedio por fruto (g); SST = sólidos soluble totales ( ${ }^{\circ}$ Brix); NL = número de lóculos.

fruto (PPF, $r=0.57$ ), longitud promedio de fruto ( $L P F, r=$ 0.57), diámetro promedio de fruto $(D P F, r=0.54)$ y total de frutos $(T F, r=0.53)$.

Lo anterior indica que los componentes del rendimiento de mayor importancia son las variables relacionadas con tamaño de fruto y número total de frutos por planta, y por ello son criterios de selección convenientes. Resultados similares obtuvieron Monamodi et al. (2013), quienes al evaluar seis líneas de jitomate de hábito determinado encontraron que el rendimiento por planta estuvo positivamente correlacionado con número de frutos por racimo $(r=0.59)$, número de racimos por planta $(r=0.87)$, número de frutos por planta $(r=0.90)$, peso de fruto por racimo $(r=0.59)$ y negativamente con sólidos soluble totales $(r=-0.41)$. De Souza et al. (2012) también encontraron que el rendimiento de frutos por planta estuvo positivamente relacionado con las variables número de frutos por planta $(r=0.94)$, peso promedio de fruto $(r=0.53)$, número de racimos por planta $(r=0.72)$ y número de frutos por racimo $(r=0.82)$.

Los sólidos soluble totales (SST) correlacionaron de manera negativa con peso total por planta (PTP, $r=-0.18$ ), peso promedio de fruto $(P P F, r=-0.30)$, longitud promedio 
Cuadro 3. Coeficientes de correlación entre10 variables de 224 genotipos derivados de una cruza interespecífica entre S. lycopersicum x S. pimpenillifolium.

\begin{tabular}{|c|c|c|c|c|c|c|c|c|c|c|}
\hline & ALT & RTP & FR & TF & PTP & PPF & LPF & DPF & SST & $\mathrm{NL}$ \\
\hline \multicolumn{11}{|l|}{ ALT } \\
\hline \multirow{2}{*}{ RTP } & $0.49 * *$ & & & & & & & & & \\
\hline & $<.0001$ & & & & & & & & & \\
\hline \multirow{2}{*}{ FR } & -0.04 & $0.22 * *$ & & & & & & & & \\
\hline & 0.37 & $<.0001$ & & & & & & & & \\
\hline \multirow{2}{*}{ TF } & 0.03 & $0.40 * *$ & 0.41 & & & & & & & \\
\hline & 0.45 & $<.0001$ & $<.0001$ & & & & & & & \\
\hline \multirow{2}{*}{ PTP } & 0.10 & 0.12 & 0.02 & $0.53 * \star$ & & & & & & \\
\hline & 0.02 & 0.00 & 0.56 & $<.0001$ & & & & & & \\
\hline \multirow{2}{*}{ PPF } & 0.01 & $-0.23 * *$ & $-0.28 * *$ & -0.09 & $0.57 * *$ & & & & & \\
\hline & 0.70 & $<.0001$ & $<.0001$ & 0.04 & $<.0001$ & & & & & \\
\hline \multirow{2}{*}{ LPF } & 0.03 & $-0.18 * *$ & $-0.29 * *$ & -0.05 & $0.57 * *$ & $0.90 * *$ & & & & \\
\hline & 0.47 & $<.0001$ & $<.0001$ & 0.23 & $<.0001$ & $<.0001$ & & & & \\
\hline \multirow{2}{*}{ DPF } & 0.01 & $-0.28 * *$ & $-0.34 * *$ & -0.10 & $0.54 * *$ & $0.92 * \star$ & $0.91 * *$ & & & \\
\hline & 0.81 & $<.0001$ & $<.0001$ & 0.0322 & $<.0001$ & $<.0001$ & $<.0001$ & & & \\
\hline \multirow{2}{*}{ SST } & 0.09 & $0.19 * *$ & 0.10 & 0.10 & $-0.18 * \star$ & $-0.30 * *$ & $-0.32 \star \star$ & $-0.36 * \star$ & & \\
\hline & 0.03 & $<.0001$ & 0.022 & 0.02 & 0.00 & $<.0001$ & $<.0001$ & $<.0001$ & & \\
\hline \multirow{2}{*}{$\mathrm{NL}$} & -0.10 & -0.13 & -0.09 & 0.00 & -0.01 & -0.01 & -0.06 & -0.01 & -0.06 & \\
\hline & 0.02 & 0.00 & 0.03 & 0.94 & 0.82 & 0.73 & 0.17 & 0.71 & 0.15 & \\
\hline
\end{tabular}

$\mathrm{ALT}=$ altura de planta $(\mathrm{cm}) ; \mathrm{RTP}=$ racimos totales por planta; FR = flores por racimo; TF = total de frutos; PTP = peso total de frutos por planta $(\mathrm{g})$; $\mathrm{PPF}=$ peso promedio por fruto $(\mathrm{g}) ; \mathrm{LPF}=$ largo promedio de fruto $(\mathrm{mm}) ; \mathrm{DPF}=$ diámetro promedio de fruto (mm); SST = sólidos solubles totales; NL = número de lóculos.

de fruto (LPF, $r=-0.32)$ y diámetro promedio de fruto (DPF, $r=-0.36)$. Esto indica que, de manera general, los componentes relacionados de manera positiva con el rendimiento de fruto por planta, como los antes mencionados, se asocian de manera negativa con la concentración de SST. Este patrón de comportamiento ha sido reportado por varios autores, como Monamodi et al. (2013) quienes encontraron una correlación negativa entre SST y rendimiento por planta, y como Georgelis y Scott (2004) quienes encontraron una correlación negativa significativa de los SST con tamaño de fruto y rendimiento, la cual atribuyeron a efectos pleiotrópicos de los genes.

A diferencia de las correlaciones negativas de SST con algunos componentes del rendimiento detectadas en el presente estudio, De Souza et al. (2012) no encontraron correlaciones significativas entre SST y las características relacionadas con tamaño de fruto y número de frutos por planta.

Las diferencias de los presentes resultados con los reportados por otros autores podrían deberse a que en este estudio se utilizaron más de 200 líneas segregantes muy diversas en su constitución genética por su origen interespecífico, mientras que en los trabajos de otros autores se utilizó material de base genética muy reducida (líneas endogámicas) y un escaso número de ellas (menos de 20 materiales genéticos).

\section{Heredabilidad}

Las variables aquí evaluadas tuvieron una heredabilidad en sentido amplio $(H)$ intermedia, de acuerdo con la escala de valores indicada por Molina (1992). Las que presentaron la $\mathrm{H}$ más alta fueron: largo de fruto (LPF), peso promedio de fruto (PPF), flores por racimo (FR) y diámetro promedio de fruto (DPF), con valores de 0.79, 0.78, 0.77 y 0.77 , respectivamente. Las variables de menor heredabilidad fueron: racimos totales por planta (RTP), sólidos solubles totales (SST) y número de lóculos (NL), todas con el valor de 0.50. Hernández-Bautista et al. (2014) estimaron valores de $\mathrm{H}$ de 0.58 para número total de frutos por planta, valor muy similar al que se encontró en este trabajo (0.62); los valores de $\mathrm{H}$ para los otros componentes de 
rendimiento (LPF, PPF y DPF) en general fueron menores a los encontrados por estos autores.

En SST, Hernández-Bautista et al. (2014) obtuvieron una $\mathrm{H}$ de 0.86 , mientras que en este trabajo se obtuvo 0.50 , atribuible a la mayor varianza fenotípica en la población segregante S3. En ocho líneas avanzadas de jitomate tipo "saladette", Gaspar-Peralta et al. (2012) encontraron valores de $\mathrm{H}$ muy bajos, en comparación con las reportadas por Hernández-Bautista et al. (2014) y con las del presente trabajo. Para los componentes del rendimiento reportadas por Gaspar-Peralta et al. (2012), las heredabilidades variaron de 0.0 a 0.7 ; por ejemplo, para rendimiento fue 0.0 mientras que en el presente trabajo fue 0.62; tal contraste probablemente se deba a que aquí se midió la H en una población de 225 líneas, con una mayor varianza genotípica que la muestra de ocho líneas avanzadas evaluadas en el estudio de Gaspar-Peralta et al. (2012).

\section{CONCLUSIONES}

La cruza interespecífica entre la especie silvestre S. pimpinellifolium y el jitomate cultivado (LOR-82) permitió generar líneas $\mathrm{S}_{3}$ con diversas combinaciones alélicas de ambos genomas, lo que originó una gran variación en cuanto a las variables relacionadas con el rendimiento y sus componentes, así como con algunas variables de calidad.

Las diferencias entre frutos de las líneas para tamaño y sólidos solubles totales permitirían que los mejoradores seleccionen las que reúnan las características deseables, pues en cuanto a tamaño y calidad de fruto hubo líneas de 1 a 36 g por fruto y de 3.4 a $10.2^{\circ}$ Brix, respectivamente. También hubo líneas con rendimientos que variaron desde 16 hasta $1330 \mathrm{~g}$ por planta, con un número de frutos por racimo de 8 a 115 .

Las variables que contribuyeron al rendimiento (peso de fruto por planta) de manera significativa fueron el peso promedio de fruto, longitud y diámetro de fruto, así como el número total de frutos por planta. Las heredabilidades en sentido amplio para componentes del rendimiento tuvieron valores mayores de 0.60, mientras que para sólidos solubles totales fue 0.50 .

\section{BIBLIOGRAFÍA}

Bonilla-Barrientos 0., R. Lobato-Ortiz, J. J. García-Zavala, S. Cruz-Izquierdo, D. Reyes-López, E. Hernández-Leal y A. Hernández-Bautista (2014) Diversidad agronómica y morfológica de tomates arriñonados y tipo pimiento de uso local en Puebla y Oaxaca, México. Revista Fitotecnia Mexicana 37:129-139.

Canady M. A. and J. R. T. Chetelat (2006) Homeologous recombination in Solanum lycopersicoides introgression lines of cultivated tomato. Genetics 174:1775-1788

Carrillo-Rodríguez J. C., J. L. Chávez-Servia, G. Rodríguez-Ortiz, R. Enríquezdel Valle y Y. Villegas-Aparicio (2013) Variación estacional de ca- racteres agromorfológicos en poblaciones nativas de jitomate (Solanum lycopersicum L.). Revista Mexicana de Ciencias Agrícolas 6:1081-1091.

Crisanto-Juárez A. U., A. M. Vera-Guzmán, J. L. Chávez-Servia y J. C. Carrillo-Rodríguez (2010) Calidad de frutos de tomates silvestres (Lycopersicon esculentum var. Cerasiforme Dunal) de Oaxaca, México. Revista Fitotecnia Mexicana 33:7-13.

De Souza L. M., P. C. T. Melo, R. R. Luders and A. M. T. Melo (2012) Correlations between yield and fruit quality characteristics of fresh market tomatoes. Horticultura Brasileira 30:627-631.

Dueñas C. F., M. C. Álvarez, C. C. Moya, C. Y. Martínez, C. B. Peteira y Y. Arias (2009) Identificación de los genes Ty-2 y Ty-3 de resistencia a Begomovirus y su grado de homocigosis en nuevas accesiones de tomate. Cultivos Tropicales 29:59-63.

Foolad R. M. (2007) Genome Mapping and Molecular Breeding of Tomato. International Journal of Plant Genomics 2007:1-52.

Gaspar-Peralta P., J. C. Carrillo-Rodríguez, J. L. Chávez-Servia, A. M. VeraGuzmán e I. Pérez-León (2012) Variación de caracteres agronómicos y licopeno en líneas avanzadas de tomate (Solanum lycopersicum L.) Revista Internacional de Botánica Experimental $81: 15-22$

Georgelis N. and J. W. Scott (2004) Relationship of tomato fruit sugar concentration with physical and chemical traits and linkage of RAPD markers. Journal of the American Society for Horticultural Science 129:839-845.

Hernández-Bautista A., R. Lobato-Ortiz, S. Cruz-Izquierdo, J. J. García-Zavala y J. L. Chávez-Servia (2014) Variación fenotípica, heterosis y heredabilidad de una cruza interespecífica de jitomate. Interciencia 39:327-332.

Hernández-Leal E., R. Lobato-Ortiz, J. J. García-Zavala, D. Reyes-López, A. Méndez-López, 0. Bonilla-Barrientos y A. Hernández-Bautista (2013) Comportamiento agronómico de poblaciones F2 de híbridos de tomate (Solanum lycopersicum L.). Revista Fitotecnia Mexicana 36:209-215.

Holland J. B., W. E. Nyquist and C. T. Cervantes-Martínez (2003) Estimating and interpreting heritability for plant breeding: an update. Plant Breeding Reviews 22:9-112.

Jenkins J. A. (1948) The origin of the cultivated tomato. Economy Botany 2:379-392.

Juárez-López, P., R. Castro-Brindis, T. Colinas-León, P. Ramírez-Vallejo, P. Sandoval-Villa, D. W. Reed, L. Cisneros-Ceballos y S. King (2009) Evaluación de la calidad en frutos de siete genotipos nativos de jitomate (Lycopersicon esculentum var. cerasiforme). Revista Chapingo Serie Horticultura 15:5-9.

Lippman Z. and S. D. Tanksley (2001) Dissecting the genetic pathway to extreme fruit size in tomato using a cross between the smallfruited wild species Lycopersicon pimpinellifolium and L. esculentum var. Giant Heirloom. Genetics 158:413-422.

Márquez S. F. (1988) Genotecnia Vegetal. Tomo II. AGT Editor. México. $665 \mathrm{p}$.

Miller J. C. and S. D. Tanksley (1990) RFLP analysis of phylogenetic relationships and genetic variation in the genus Lycopersicon. Theoretical and Applied Genetics 80:437-448.

Molina G. J. D. (1992) Introducción a la Genética de Poblaciones y Cuantitativa. AGT Editor. México. 349 p.

Monamodi E. L., D. M. Lungu and G. L. Fite (2013) Analysis of fruit yield and its components in determinate tomato (Lycopersicon lycopersci) using correlation and path coefficient. Botswana Journal of Agriculture and Applied Sciences 9:29-40.

Peralta I. E. and D. M. Spooner (2007) History, Origin and Early Cultivation of Tomato (Solanaceae). In: Genetic Improvement of Solanaceous Crop, Vol. 2: Tomato. M. K. Razdan and A. K. Mattoo (eds.). Science Publishers. Enfield, New Hampshire, USA. pp:124.

Peralta I. E., D. M. Spooner and S. Knapp (2008) Taxonomy of Wild Tomatoes and their Relatives (Solanum sections Lycopersicoides, Juglandifolia, Lycopersicon; Solanaceae). Systematic Botany Monographs. American Society of Plant Taxonomists (eds.) Michigan, USA. $186 \mathrm{p}$

Rick C. M., and J. F. Forbes (1975) Allozyme variation in the cultivated tomato and closely related species. Bulletin of the Torrey Botanical Club 102:376-384

SAS Institute (2002) User's Guide of SAS (Statistical Analysis System). SAS Institute Inc. Cary, N. C. USA. 550 p. 
SIAP, Sistema de Información Agroalimentaria y Pesquera (2015) Producción Agropecuaria y Pesquera. http://www.siap.gob.mx/. (Diciembre 2015).
Steiner A. A. (1984) The Universal Nutrient Solution. In: Proceedings 6th International Congress on Soilless Culture. Wageningen, The Netherlands. pp: 633-650. 